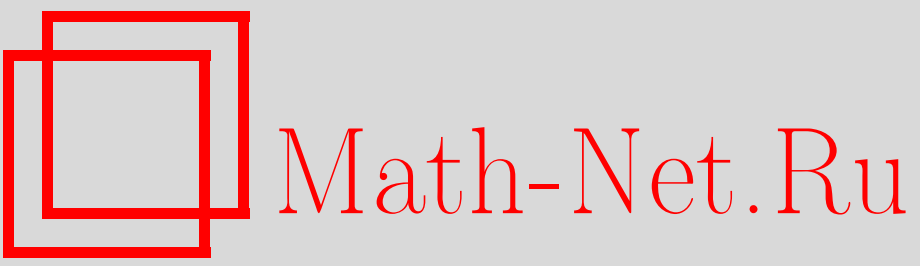

В. В. Козлов, Д. В. Трещёв, Слабая сходимость решений уравнения Лиувилля для нелинейных гамильтоновых систем, ТМФ, 2003, том 134, номер 3, 388-400

DOI: https://doi.org/10.4213/tmf164

Использование Общероссийского математического портала Math-Net.Ru подразумевает, что вы прочитали и согласны с пользовательским соглашением

http://www.mathnet.ru/rus/agreement

Параметры загрузки:

IP: 3.89 .185 .249

26 апреля 2023 г., 17:42:19 
ТЕОРЕТИЧЕСКАЯ

И МАТЕМАТИЧЕСКАЯ

ФИЗИКА

Том 134, № 3

март, 2003

(C) 2003 г.

В.В. Козлов*, Д.В. Трешев*

\section{СЛАБАЯ СХОДИМОСТЬ РЕШЕНИЙ УРАВНЕНИЯ ЛИУВИЛЛЯ ДЛЯ НЕЛИНЕЙНЫХ ГАМИЛЬТОНОВЫХ СИСТЕМ}

Предложены достаточные условия существования слабого предела решений уравнений Лиувилля при неограниченном возрастании времени. Наличие слабого предела плотности распределения вероятностей приводит к новой интерпретации второго закона термодинамики о росте энтропии.

Ключевые слова: гамильтонова система, уравнение Лиувилля, слабая сходимость, энтропия.

\section{1. ВВЕДЕНИЕ}

Пусть $\Gamma=T^{*} M-$ фазовое пространство автономной гамильтоновой системы, $M=$ $\left\{x_{1}, \ldots, x_{n}\right\}-$ конфигурационное пространство, $H(x, y)$ - функция Гамильтона, $y=$ $\left(y_{1}, \ldots, y_{n}\right)$ - канонические импульсы.

Согласно Гиббсу [1], в начальный момент времени $t=0$ в фазовом пространстве $\Gamma$ вводится вероятностная мера $\mu(\mu(\Gamma)=1)$. Предположим, что мера $\mu$ имеет плотность $\rho(x, y)$. Ясно, что $\rho$ - функция из класса $L_{1}(\Gamma)$. Эта мера переносится фазовым потоком $g^{t}$ гамильтоновой системы. Поэтому ее плотность $\rho_{t}$ зависит от времени и удовлетворяет уравнению Лиувилля: если $\rho_{t} \in C^{1}(\Gamma)$, то

$$
\frac{\partial \rho_{t}}{\partial t}+\sum_{i=1}^{n}\left[\frac{\partial}{\partial x_{i}}\left(\rho_{t} \frac{\partial H}{\partial y_{i}}\right)-\frac{\partial}{\partial y_{i}}\left(\rho_{t} \frac{\partial H}{\partial x_{i}}\right)\right]=0 .
$$

Функция $\rho_{0}=\rho$ будет данным Коши.

Уравнение Лиувилля играет ключевую роль во всей статистической механике. Достаточно упомянуть цепочку уравнений Боголюбова [2] (обзор развития идей Боголюбова см., например, в [3]). Однако при этом возникает ряд затруднений принципиального характера. Во-первых, каким образом следует выбирать начальную плотность распределения вероятностей $\rho_{0}$ ? Во-вторых, имеет ли функция $\rho_{t}$ предел при $t \rightarrow \pm \infty$ ?

Вопрос о выборе $\rho_{0}$ подробно обсуждается в монографиях [2], [3]. Что касается второго вопроса, то еще Гиббс пытался показать, что при $t \rightarrow \infty$ плотность $\rho_{t}$ стремится

\footnotetext{
* Московский государственный университет, Москва, Россия
} 
(в каком-то смысле) к плотности стационарного распределения, которое отвечает состоянию теплового равновесия. С этой целью он ввел микроканоническое распределение вероятностей, когда плотность зависит лишш от полной энергии $H$. Однако согласно теореме Пуанкаре о возврашении, как правило, $\rho_{t}$ вообше не имеет предела при $t \rightarrow \pm \infty$ в обычном смысле.

Мы будем изучать вопрос о слабой сходимости решений уравнения Лиувилля. Такой подход естествен с точки зрения обоснования термодинамики - перехода к макроскопическому описанию эволюции динамической системы, поскольку плотность вероятностной меры “существует” не сама по себе, а проявляется при вычислении средних значений (математических ожиданий) динамических величин. Такая точка зрения в неявной форме использовалась еше Пуанкаре в работе [4] (подробнее об этом в разделе 6), которая оказалась не понятой и не востребованной специалистами по статистической механике.

Ограничимся более узким классом начальных распределений и будем считать $\rho$ функцией, интегрируемой со своим квадратом (из $L_{2}(\Gamma)$ ). Это предположение естественно с точки зрения возможности вычисления средних значений функций, заданных на фазовом пространстве. Пусть $z$ - точка фазового пространства Г (она задается набором канонических переменных $x, y),\left\{g^{t}\right\}$ - фазовый поток гамильтоновой системы с гамильтонианом $H$. Будем считать, что все решения этой гамильтоновой системы продолжимы на всю ось времени $\mathbb{R}=\{t\}$. Например, можно предполагать, что все энергетические многообразия $\{H=$ const $\} \subset \Gamma$ компактны. В этом случае преобразования $g^{t}$ определены для всех $t \in \mathbb{R}$.

Так как преобразования $g^{t}$ сохраняют фазовый объем в Г (теорема Лиувилля), то $\rho_{t}$ - первый интеграл уравнений Гамильтона с гамильтонианом $H$. Это простое обстоятельство позволяет указать общий вид решений уравнения Лиувилля. Пусть $z_{0}-$ начальное состояние системы. Тогда $t \mapsto g^{t}\left(z_{0}\right)$ - решение уравнений Гамильтона. Любой первый интеграл - функция от начальных данных: $\rho\left(z_{0}\right)$. Поскольку $z_{0}=g^{-t}(z)$, то

$$
\rho_{t}(z)=\rho\left(g^{-t}(z)\right)
$$

Хорошо известно, что перенос функций из $L_{2}$ фазовым потоком динамической системы с инвариантной мерой эквивалентен действию однопараметрической группы унитарных операторов $U^{t}$ (см., например, [5]): $U^{t} \rho(z)=\rho\left(g^{t}(z)\right)$, так что $\rho_{t}=U^{-t} \rho$. Оператор $U$ часто называют оператором Купмана.

Пусть $\varphi$ - еше одна функция из $L_{2}(\Gamma)$. Тогда корректно определена функция времени

$$
K(t)=\left(U^{-t} \rho, \varphi\right)=\int_{\Gamma} \varphi U^{-t} \rho d^{2 n} z
$$

Она имеет простой смысл: если $\varphi$ - характеристическая функция некоторой измеримой области $\Phi$ в $\Gamma$, то $K(t)$ - доля гамильтоновых систем из ансамбля Гиббса, находяшихся в области $\Phi$ в момент времени $t$. Если

$$
\lim _{t \rightarrow \infty} K(t)=(\bar{\rho}, \varphi)
$$

для любой $\varphi \in L_{2}$, то $\rho_{t}$ слабо сходится к функции $\bar{\rho}$. 
Наша цель - указать условия слабой сходимости и способ вычисления слабых пределов плотностей вероятностных мер.

Настоящая работа развивает и дополняет результаты статьи [6]. В разделе 2 будет указана формула для слабого предела $\bar{\rho}$ плотности распределения вероятностей $\rho_{t}$ при условии, что он сушествует. В разделе 3 выделен класс систем (вида (3.1)), которые являются основным объектом изучения. Приведены необходимые мотивировки и примеры, а также обсуждается основной технический аппарат для дальнейшего анализа - обобщение статистической эргодической теоремы фон Неймана, когда усреднение по времени заменяется усреднением по некоторой вероятностной мере. В четвертом разделе указан класс систем (с так называемыми слоистыми потоками), для которых удается доказать слабую сходимость решений уравнения Лиувилля при неограниченном возрастании времени. Типичными представителями этого класса динамических систем являются геодезические потоки и другие гамильтоновы квазиоднородные системы. Доказательства основных результатов приведены в разделе 5 . В заключительном разделе 6 доказывается, что энтропия предельной меры $\bar{\rho} d^{2 n} z$ не меньше энтропии исходной меры $\rho d^{2 n} z$, а также обсуждается связь этого результата со вторым началом термодинамики.

\section{2. СЛАБЫЙ ПРЕДЕЛ}

Теорема 1. Пусть для некоторой функции $\varphi \in L_{2}(\Gamma)$ существует предел

$$
\lim _{t \rightarrow \infty} K(t)=K_{\infty} .
$$

Тогда $K_{\infty}=(\bar{\rho}, \varphi)$, əде

$$
\bar{\rho}(z)=\lim _{T \rightarrow \infty} \frac{1}{T} \int_{0}^{T} \rho\left(g^{t}(z)\right) d t .
$$

Равенство (1) имеет место с точностью до множества точек $z \in$ Г меры нуль (как это вообще принято в теории меры). Так как $\rho \in L_{1}$, то (по теореме Биркгофа-Хинчина) $\bar{\rho}$ определена почти всюду, неотрицательна, является интегралом уравнений Гамильтона (инвариантна относительно $g^{t}$ ) и (если энергетические поверхности $H=$ const компактны)

$$
\int_{\Gamma} \bar{\rho} d^{2 n} z=1 .
$$

Следовательно, $\bar{\rho}$ является плотностью стационарной вероятностной меры.

Если предел $\lim K(t)$ сушествует для любой функции $\varphi \in L_{2}$, то функция $\bar{\rho}$, удовлетворяюшая равенству $K_{\infty}=(\bar{\rho}, \varphi)$, единственна. Отсюда вытекает

СледСтвиЕ 1. Если $\rho_{t}$ слабо сходится $\kappa \bar{\rho}$, то $\bar{\rho}$ определяется равенством (1).

ДоКАЗАТЕЛЬСТво теОРемЫ 1 . Пусть $\left(\rho_{t}, \varphi\right) \rightarrow K_{\infty}$ при $t \rightarrow \infty$. Тогда (по теореме Коши)

$$
\frac{1}{T} \int_{0}^{T}\left(\rho_{t}, \varphi\right) d t \rightarrow K_{\infty}
$$


при $T \rightarrow \infty$. Стоит отметить, что интеграл слева в этом соотношении сушествует при всех $T$, если $\rho$ и $\varphi$ - функции из $L_{2}$ (см., например, [7]). По теореме Фубини

$$
\frac{1}{T} \int_{0}^{T}\left(\rho_{t}, \varphi\right) d t=\int_{\Gamma} \tilde{\rho}_{T}(z) \varphi(z) d^{2 n} z
$$

где

$$
\tilde{\rho}_{T}=\frac{1}{T} \int_{0}^{T} \rho\left(g^{t}(z)\right) d t
$$

Далее по теореме фон Неймана

$$
\int_{\Gamma}(\tilde{\rho}-\bar{\rho}) d^{2 n} z \rightarrow 0
$$

при $T \rightarrow \infty$. Отсюда вытекает, что

$$
\frac{1}{T} \int_{0}^{T}\left(\rho_{t}, \varphi\right) d t \rightarrow(\bar{\rho}, \varphi)
$$

Действительно, с учетом теоремы фон Неймана

$$
\left[\int_{\Gamma}\left(\tilde{\rho}_{T}-\bar{\rho}\right) \varphi d^{2 n} z\right]^{2} \leqslant \int_{\Gamma}\left(\tilde{\rho}_{T}-\bar{\rho}\right)^{2} d^{2 n} z \int_{\Gamma} \varphi^{2} d^{2 n} z \rightarrow 0
$$

когда $T \rightarrow \infty$. Что и требовалось доказать.

Теорема 1 носит априорный характер: если слабый предел плотности вероятностей сушествует, то он представляется хорошо известным объектом эргодической теории.

Вопрос теперь сводится к отысканию условий, при которых плотность $\rho_{t}$ имеет слабый предел при $t \rightarrow \pm \infty$. Отметим, что слабая сходимость имеет место не всегда. Исключение составляют, например, линейные гамильтоновы системы.

ЗАмЕЧАниЕ 1. Чезаровское среднее (1) можно заменить средними более общего вида. Например, можно положить

$$
\bar{\rho}(z)=\lim _{T \rightarrow \infty}\left(\int_{0}^{T} \alpha(t) \rho\left(g^{t}(z)\right) d t / \int_{0}^{T} \alpha(t) d t\right)
$$

где $\alpha(t)>0$ и интеграл

$$
\int_{0}^{\infty} \alpha(t) d t
$$

расходится. Если предел (2) сушествует, то он совпадает с (1) [8]. 


\section{3. ОБОБЩЕНИЕ СТАТИСТИЧЕСКОЙ ЭРГОДИЧЕСКОЙ ТЕОРЕМЫ}

Вопрос о сушествовании слабого предела плотности $\rho_{t}$ рассмотрим для динамических систем, которые задаются дифференциальными уравнениями вида

$$
\dot{z}=v(z, \omega), \quad \dot{\omega}=0 .
$$

Фазовым пространством Г является прямое произведение $\Lambda \times D$, где $\Lambda=\left\{z_{1}, \ldots, z_{n}\right\}-$ гладкое $n$-мерное многообразие, а $D$ - область в $\mathbb{R}^{m}=\left\{\omega_{1}, \ldots, \omega_{m}\right\}$. Координаты $\omega$ будут первыми интегралами. Будем предполагать, что при фиксированных $\omega$ система на $\Lambda$ имеет инвариантную меру $d \nu=\lambda(z, \omega) d^{2 n} z$ :

$$
\sum \frac{\partial\left(v_{i} \lambda\right)}{\partial z_{i}}=0
$$

Такой вид имеют, в частности, гамильтоновы системы. Здесь $m=1$, а $\Lambda$ - энергетическая поверхность. Роль координаты $\omega$ играет полная энергия. Фазовое пространство Г гамильтоновой системы разбивается на клетки $h_{1} \leqslant H \leqslant h_{2}$, причем в интервале $\left(h_{1}, h_{2}\right)$ нет критических значений функции Гамильтона $H$.

Рассмотрим сначала частный случай уравнений $(3)$, когда поле $v(z, \omega)$ имеет вид произведения $\omega v(z)$, где $z \mapsto v(z)$ - некоторое гладкое векторное поле на многообразии $\Lambda$. Фазовый поток такой системы - семейство преобразований $\left\{g^{\omega t}\right\}$, где $\left\{g^{t}\right\}-$ поток динамической системы

$$
\dot{z}=v(z), \quad z \in \Lambda \text {. }
$$

Ее инвариантная мера $\nu$ не зависит от параметра $\omega$.

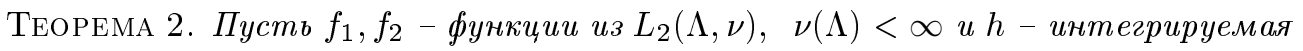
функиия на измеримом множсестве $I \subset \mathbb{R}=\{\omega\}\left(\right.$ из $\left.L_{1}(I, d \omega)\right)$. Тогда

$$
\lim _{t \rightarrow \infty} \int_{I} h(\omega)\left(U^{\omega t} f_{1}, f_{2}\right) d \omega=\left(\bar{f}_{1}, f_{2}\right) \int_{I} h(\omega) d \omega .
$$

Теорема 2 содержит как частный случай классическую теорему фон Неймана [7]. Действительно, пусть $h$ - характеристическая функция отрезка [0, 1]. Тогда для любой интегрируемой функции $\varphi: \mathbb{R} \rightarrow \mathbb{R}$

$$
\int_{-\infty}^{+\infty} h(\omega) \varphi(\omega t) d \omega=\frac{1}{t} \int_{0}^{t} \varphi(s) d s .
$$

Особенно просто формула (4) выглядит в случае эргодического потока. Если $h-$ плотность вероятностной меры на $\mathbb{R}=\{\omega\}$, то

$$
\lim _{t \rightarrow \infty} \int_{-\infty}^{+\infty} \int_{\Lambda} h(\omega) f_{1}\left(g^{\omega t}(x)\right) f_{2}(x) d \nu d \omega=\nu(\Lambda) \int_{\Lambda} f_{1} d \nu \int_{\Lambda} f_{2} d \nu
$$

Таким образом, в среднем при больших значениях $t$ функции $f_{1}\left(g^{\omega t}(x)\right)$ и $f_{2}(x)$ статистически независимы: интеграл от произведения равен произведению интегралов. Частные случаи формулы (5) указаны нами ранее в работе [6]. 
Укажем еще одно любопытное следствие формулы (5): когда дисперсия $\sigma$ нормального распределения неограниченно возрастает,

$$
\frac{1}{\sqrt{2 \pi} \sigma} \int_{-\infty}^{+\infty} \int_{\Lambda} e^{-t^{2} / 2 \sigma^{2}} f_{1}\left(g^{t}(x)\right) f_{2}(x) d \nu d t \rightarrow \nu(\Lambda) \int_{\Lambda} f_{1} d \nu \int_{\Lambda} f_{2} d \nu
$$

Доказательство теоремы 2 само использует эргодическую теорему фон Неймана. Для любого $\varepsilon>0$ найдется кусочно-постоянная функция $h_{\varepsilon}: \mathbb{R} \rightarrow \mathbb{R}$ такая, что

1) $h_{\varepsilon}(\omega)=c_{k}=$ const на интервалах $\left(\omega_{k}, \omega_{k+1}\right), k=1, \ldots, N$ (возможно, что $\omega_{1}=$ $-\infty$ и $\left.\omega_{N+1}=+\infty\right)$,

2) $I \subset\left(\omega_{1}, \omega_{N+1}\right)$,

3) $\int_{I}\left|h-h_{\varepsilon}\right| d \omega<\varepsilon$.

Тогда (с учетом изометричности оператора $U$ )

$$
\begin{gathered}
\left|\int_{I} h(\omega)\left(U^{\omega t} f_{1}, f_{2}\right) d \omega-\int_{I} h_{\varepsilon}(\omega)\left(U^{\omega t} f_{1}, f_{2}\right) d \omega\right| \leqslant \\
\leqslant \int_{I}\left|h-h_{\varepsilon}\right| d \omega\left\|f_{1}\right\|\left\|f_{2}\right\| \leqslant \varepsilon\left\|f_{1}\right\|\left\|f_{2}\right\|,
\end{gathered}
$$

где $\|\cdot\|$ - норма в $L_{2}$. Таким образом, достаточно установить сходимость интегралов

$$
J_{k}(t)=\int_{\omega_{k}}^{\omega_{k+1}} h_{\varepsilon}(\omega)\left(U^{\omega t} f_{1}, f_{2}\right) d \omega
$$

По теореме фон Неймана

$$
J_{k}(t)=\frac{c_{k}}{t} \int_{\omega_{k} t}^{\omega_{k+1} t}\left(U^{s} f_{1}, f_{2}\right) d s \rightarrow c_{k}\left(\omega_{k+1}-\omega_{k}\right)\left(\bar{f}_{1}, f_{2}\right)
$$

при $t \rightarrow \infty$. Остается заметить, что

$$
\sum_{1}^{N} c_{k}\left(\omega_{k+1}-\omega_{k}\right)=\int_{I} h_{\varepsilon}(\omega) d \omega=\int_{I} h(\omega) d \omega+\delta
$$

причем $|\delta| \leqslant \varepsilon$. Что и требовалось доказать.

ЗАмЕчАниЕ 2. При первоначальном доказательстве теоремы 2 (и основной теоремы 3 из раздела 4) мы использовали формулу Стоуна спектрального разложения группы унитарных операторов, как это было намечено в работе [6]. Отметим, что исходным пунктом доказательства фон Неймана статистической эргодической теоремы также является формула Стоуна (см., например, [9]). Современные доказательства эргодических теорем используют другую технику. 


\section{4. ПРЕДЕЛЬНЫЕ МЕРЫ СЛОИСТЫХ ПОТОКОВ}

Применим метод, использованный в предыдущем разделе, к динамическим системам со слоистыми фазовыми потоками. Эти системы являются частным случаем систем (3), когда имеется всего одна переменная $\omega(m=1)$. Их выделяет следуюшее характерное свойство: фазовые потоки на $\Lambda$ при различных значениях $\omega$ оказываются сопряженными (после подходяшей замены времени). Важным примером слоистых потоков служат геодезические потоки на гладких многообразиях. Перейдем к точным определениям.

Пусть $I$ - интервал числовой прямой $\mathbb{R}$ (возможно, бесконечный), $\Lambda$ - гладкое многообразие. Пусть в фазовом пространстве $\Gamma=\Lambda \times I$ задана динамическая система (3), где $z \in \Lambda, \omega \in I$.

Положим $P_{\gamma}=\{(z, \omega) \in \Gamma: \omega=\gamma\}$. Это $n$-мерные интегральные многообразия системы $(3)$. Отображение $\psi_{\omega}:(z, \omega) \rightarrow z$ задает естественный диффеоморфизм $P_{\omega}$ и $\Lambda$. Ясно, что векторное поле $v$ рассматриваемой динамической системы касается $P_{\omega}$ в точ$\operatorname{kax}(z, \omega) \in \Gamma$. Обозначим $v_{\omega}$ ограничение поля $v$ на $P_{\omega}$.

Далее, пусть $\left\{g^{t}\right\}$ - фазовый поток на $\Gamma$, порождаемый системой $(3)$, а $\left\{g_{\omega}^{t}\right\}$ - его ограничение на $P_{\omega}$. Поскольку все многообразия $P_{\omega}$ диффеоморфны $\Lambda$, то можно считать, что $\left\{g_{\omega}^{t}\right\}$ - однопараметрическое семейство групп преобразований $\Lambda$ ( $\omega$ - параметр семейства).

ОПРЕДЕЛЕНИЕ 1 . Поток $g^{t}$ назовем слоистьм. , если сушествуют гладкая функция $\alpha: I \rightarrow(0, \infty)$ и поток $g_{*}^{s}: \Lambda \rightarrow \Lambda$ такие, что диаграмма

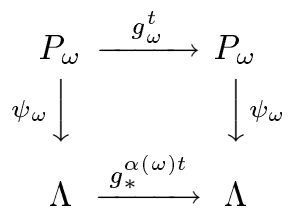

коммутативна для всех $\omega \in I$ и всех $t \in \mathbb{R}$. Слоистый поток назовем невырожденным, если функция $\omega \mapsto \alpha(\omega)$ имеет лиш изолированные критические точки.

Отождествляя $P_{\omega}$ и $\Lambda$ с помощью диффеоморфизма $\psi_{\omega}$, свойство коммутативности диаграммы (6) можно представить в виде следуюшего равенства:

$$
g_{\omega}^{t}=g_{*}^{\alpha(\omega) t}
$$

Имеет место очевидное

ПРЕДЛОЖЕНИЕ 1. Пусть поток $g_{*}^{t}$ сохраняет меру $\nu_{*}$ на многообразии $\Lambda$ и любая мера на интервале $I$. Тогда поток $g^{t}$ на $\Lambda \times I$ сохраняет меру $\mu=\nu_{*} \times \sigma$.

Прокомментируем это утверждение в случае, когда меры $\nu_{*}$ и $\sigma$ имеют гладкие плотности:

$$
d \nu_{*}=\lambda(z) d^{n} z, \quad d \sigma=\varphi(\omega) d \omega .
$$

Пусть поток $g_{*}^{t}$ порождает векторное поле на $\Lambda$

$$
v_{*}(z)=\left.\frac{d}{d t}\right|_{t=0}\left(g_{*}^{t}(z)\right),
$$


так что поток $g_{*}^{t}$ будет фазовым потоком системы дифференциальных уравнений на $\Lambda$ : $\dot{z}=v_{*}(z)$. Условие (7) означает, что для слоистого потока система (3) имеет следуюший вид:

$$
\dot{z}=\alpha(\omega) v_{*}(z), \quad \dot{\omega}=0 .
$$

Предложение об инвариантности меры $v_{*}$ относительно действия потока $g_{*}^{t}$ означает, что ее плотность $\lambda$ удовлетворяет уравнению Лиувилля

$$
\sum_{i=1}^{n} \frac{\partial \lambda\left(v_{*}\right)_{i}}{\partial z_{i}}=0
$$

Следовательно, плотность $\rho=\lambda(z) \varphi(\omega)$ меры $\mu$ удовлетворяет уравнению

$$
\sum_{i=1}^{n} \frac{\partial \rho v_{i}}{\partial z_{i}}=0
$$

где $v=\lambda v_{*}$. С учетом равенства $\dot{\omega}=0$ это соотношение - критерий инвариантности меры $\mu$ относительно фазового потока системы (8).

Пусть $U^{t}$ - семейство унитарных операторов Купмана на $L_{2}(\Gamma, \mu)$, порождаемых потоком $g^{t}$.

ТЕОРема 3. Пусть $g^{t}-$ невырожденный слоистый поток на $\Gamma=\Lambda \times I$, мера $\nu_{*}$ абсолютно непрерьвна относительно мерь, задаваемой какой-нибудь римановой метрикой на $\Lambda$, мера $\sigma$ абсолютно непрерывна относительно обычной меры Лебега на $\mathbb{R}$, причем $\nu_{*}(\Lambda)=\sigma(I)=1$. Тогда для любих $f^{\prime}, f^{\prime \prime} \in L_{2}(\Gamma, \mu)$ существует предел $\lim _{t \rightarrow \infty}\left(U^{t} f^{\prime}, f^{\prime \prime}\right)$.

Из теорем 1 и 3 вытекает

СлеДСТвИЕ 2. Для динамических систем вида (3) с невырожсенным слоистым фазовым потоком плотность распределения вероятностей $\rho_{t}$ имеет слабье предель при $t \rightarrow+\infty u t \rightarrow-\infty$, причем эти предель совпадают и вычисляются по формуле (1).

Теорема 3 доказывается в следуюшем разделе. Прежде чем приступить к ее доказательству, приведем некоторые примеры динамических систем со слоистыми фазовыми потоками.

Пусть $P_{\omega}, \omega>0,-$ однопараметрическое семейство гладких многообразий, $\varphi_{\omega}: P_{1} \rightarrow$ $P_{\omega}$ - семейство диффеоморфизмов. Объединение $\Gamma=\bigcup_{\omega>0} P_{\omega}$ имеет структуру прямого произведения $\Lambda \times I$, где $\Lambda=P_{1}$, а $I$ - бесконечный интервал $(0,+\infty)$. Снабдим $\Gamma$ гладкой структурой прямого произведения $\Lambda \times I$.

ОПРЕДЕЛЕНИЕ 2 . Векторное поле $v$ на Г назовем $\varphi_{\omega}$-однородным степени $k$, если для некоторого векторного поля $\varphi_{1}$ на $P_{1}$

$$
v \circ \varphi_{\omega}=\omega^{k}\left(D \varphi_{\omega}\right) v_{1}
$$

при всех $\omega>0$. Здесь $D$ - дифференщиал отображения.

Поскольку $v_{1}$ касается $P_{1}$ и $\varphi_{\omega}\left(P_{1}\right)=P_{\omega}$, то поле $v$ касательно к слоям $P_{\omega}, \omega>0$. 
Пример 1. Пусть $(M,\langle\rangle$,$) - гладкое риманово многообразие, \langle,\rangle^{*}$ - метрика, сопряженная метрике $\langle$,$\rangle . Пусть p$ - элемент сопряженного пространства $T_{q}^{*} M$ (импульс механической системы). Положим $\|p\|^{2}=\langle p, p\rangle^{*}$ и $P_{s}=\left\{p \in T_{q}^{*} M: q \in M,\|p\|=s\right\}$. Ясно, что $\Gamma=T^{*} M \backslash P_{0}$.

Пусть $v$-гамильтоново векторное поле на $\Gamma$, заданное стандартной симплектической структурой $\sum d p_{i} \wedge d q_{i}$ и гамильтонианом $H=\|p\|^{2} / 2$. Это поле порождает динамическую систему на $\Gamma$, которая называется геодезическим потоком. Соответствуюший фазовый поток $g^{t}$ определен при всех $t \in \mathbb{R}$, если $(M,\langle\rangle$,$) - полное риманово многооб-$ разие (все геодезические имеют бесконечную длину).

Пусть $(q, p)$ - точка из Г. Положим $\varphi_{\omega}(q, p)=(q, \omega p), \omega>0$. Тогда гамильтоново векторное поле $v$, задаваемое уравнениями Гамильтона

$$
\dot{q}=\frac{\partial H}{\partial p}, \quad \dot{p}=-\frac{\partial H}{\partial q}
$$

будет $\varphi_{\omega}$-однородным степени $k=1$. Действительно, структура уравнений геодезических (10) такова, что $\dot{p}$ квадратично по $p$, а $\dot{q}$ линейно по $p$.

ПримеР 2. Рассмотрим в $\mathbb{R}^{2 n}=\left\{q_{1}, \ldots, q_{n}, p_{1}, \ldots, p_{n}\right\}$ гамильтонову систему (10) с гамильтонианом

$$
H=\frac{1}{2} \sum_{i=1}^{n}\left(p_{i}^{2}+\alpha_{i}^{2} q_{i}^{2}\right), \quad \alpha_{i}>0
$$

(полигармонический осциллятор с частотами $\alpha_{1}, \ldots, \alpha_{n}$ ). Положим снова

$$
P_{\omega}=\left\{(p, q) \in \mathbb{R}^{2 n}: H(p, q)=\omega\right\}, \quad \omega>0,
$$

и $\varphi_{\omega}:(q, p) \rightarrow(\omega q, \omega p)$. Легко понять, что гамильтоново векторное поле $v$ на $\Gamma=$ $\bigcup_{\omega>0} P_{\omega}$, порождаемое квадратичным гамильтонианом $(11)$, будет $\varphi_{\omega}$-однородным нулевой степени $(k=0)$.

ПРЕДЛОЖЕНИЕ 2. Пусть $v$ является $\varphi_{\omega}$-однородным векторным полем на Г степени $k$. Тогда $v$ порождает на Г слоистый поток $g^{t}$, причем $\alpha(\omega)=\omega^{k}$.

СЛЕДСТВИЕ 3. При $k \neq 0$ поток $g^{t}$ невырожден.

Таким образом, геодезический поток невырожден, а потоки, порождаемые линейными гамильтоновыми системами (пример 2), вырожденны.

ДОКАЗАТЕЛЬСТВО ПРЕДЛОЖЕНИЯ 2 . ПоЛОЖим $\Lambda=P_{1}$ и Заметим, что Любую точку $z \in \Gamma$ можно однозначно представить в виде

$$
z=\varphi_{\omega}\left(z_{1}\right), \quad \omega>0, \quad z_{1} \in P_{1} .
$$

Так как отображение $\Lambda \times(0,+\infty) \rightarrow \Gamma$, задаваемое равенством (12), является диффеоморфизмом, то достаточно проверить, что

$$
g^{t} \circ \varphi_{\omega}=\varphi_{\omega} \circ g_{1}^{\omega^{k} t},
$$

где $g_{1}^{t}$ - ограничение потока $g^{t}$ на $P_{1}$. 
Это равенство, очевидно, справедливо при $t=0$. Проверим равенство производных по $t$. Диффференцируя по $t$ при $t=0$, получаем

$$
\begin{aligned}
\left.\frac{d}{d t}\right|_{t=0} g^{t} \circ \varphi_{\omega} & =v \circ \varphi_{\omega}, \\
\left.\frac{d}{d t}\right|_{t=0} \varphi_{\omega} \circ g_{1}^{\omega^{k} t} & =\omega^{k}\left(D \varphi_{v}\right) v_{1},
\end{aligned}
$$

где $v_{1}$ - ограничение поля $v$ на $P_{1}$. Равенство этих производных вытекает из (9) - определения $\varphi_{\omega}$-однородного поля степени $k$. Равенство производных при всех значениях $t$ вытекает из группового свойства фазовых потоков. Предложение доказано.

\section{5. ОПЕРАТОР КУПМАНА ДЛЯ СЛОИСТЫХ ПОТОКОВ}

В этом параграфе мы докажем теорему 3 . Пусть $U^{t}, U_{\omega}^{t}, U_{*}^{t}-$ унитарные операторы Купмана, отвечающие потокам $g^{t}, g_{\omega}^{t}, g_{*}^{t}$, соответственно.

ПРЕДЛОЖЕНИЕ 3. Справедливо равенство

$$
U_{\omega}^{t}=U_{*}^{\alpha(\omega) t}
$$

Действительно, для произвольной функции $f$ из $L_{2}\left(\Lambda, \nu_{*}\right)$ имеем

$$
U_{\omega}^{t} f=f \circ g_{\omega}^{t}=f \circ g_{*}^{\alpha(\omega) t}=U_{*}^{\alpha(\omega) t} f .
$$

При выводе второго равенства в этой цепочке мы отождествили многообразия $\Lambda$ и $P_{\omega}$ с помошью диффеоморфизма $\psi_{\omega}$ и затем воспользовались равенством $(7)$. Предложение доказано.

Пусть $f^{\prime}$ и $f^{\prime \prime}$ - функции из $L_{2}(\Gamma, \mu), \Gamma=\Lambda \times I$. Положим

$$
f_{\omega}^{\prime}(\cdot)=f^{\prime}(\cdot, \omega), \quad f_{\omega}^{\prime \prime}(\cdot)=f^{\prime \prime}(\cdot, \omega) .
$$

Применяя теорему Фубини и предложение 3, получим

$$
\left(U^{t} f^{\prime}, f^{\prime \prime}\right)=\int_{I}\left(U_{\omega}^{t} f_{\omega}^{\prime}, f_{\omega}^{\prime \prime}\right) d \sigma=\int_{I}\left(U_{*}^{\alpha(\omega) t} f_{\omega}^{\prime}, f_{\omega}^{\prime \prime}\right) d \sigma .
$$

Пусть $A^{\prime}, A^{\prime \prime}$ - измеримые подмножества $\Lambda$, а $I^{\prime}, I^{\prime \prime}$ - интервалы в $I$. Пусть $\chi^{\prime}, \chi^{\prime \prime}$ : $\Gamma \rightarrow \mathbb{R}$ - характеристические функции (индикаторы) множеств $A^{\prime} \times I^{\prime}, A^{\prime \prime} \times I^{\prime \prime}$, соответственно. Положим $J(t)=\left(U^{t} \chi^{\prime}, \chi^{\prime \prime}\right)$.

ЛЕмма (основная). Предель $\lim _{t \rightarrow+\infty} J(t) u \lim _{t \rightarrow-\infty} J(t)$ существуют.

Теорема 3 вытекает из основной леммы. Действительно, так как $\nu_{*}$ абсолютно непрерывна относительно меры, задаваемой на $\Lambda$ некоторой римановой метрикой, и $\sigma$ абсолютно непрерывна относительно меры Лебега, то в $L_{2}(\Gamma, \mu)$ пространство непрерывных на Г функций с компактным носителем всюду плотно. В свою очередь в этом пространстве всюду плотно (даже в $C^{0}$-норме) линейное пространство функций, которые являются линейными комбинациями индикаторов $\mu$-измеримых подмножеств из $Г$. 
Пусть теперь $f^{\prime}$ и $f^{\prime \prime}$ - любые две функции из $L_{2}(\Gamma, \mu)$. Для доказательства существования предела $\lim _{t \rightarrow+\infty}\left(U^{t} f^{\prime}, f^{\prime \prime}\right)$ воспользуемся критерием Коши: надо показать, что разность

$$
\left(U^{t_{1}} f^{\prime}, f^{\prime \prime}\right)-\left(U^{t_{2}} f^{\prime}, f^{\prime \prime}\right)
$$

меньше любого заданного $\varepsilon>0$ для всех $t_{1}, t_{2}>T(\varepsilon)$. Для этого аппроксимируем $f^{\prime}$ и $f^{\prime \prime}$ функциями $g^{\prime}$ и $g^{\prime \prime}$, которые являются конечными линейными комбинациями индикаторов $\chi^{\prime}$ и $\chi^{\prime \prime}:$ для любого $\varepsilon>0$ найдутся функции $g^{\prime}$ и $g^{\prime \prime}$ такие, что

$$
\left\|f^{\prime}-g^{\prime}\right\|<\varepsilon, \quad\left\|f^{\prime \prime}-g^{\prime \prime}\right\|<\varepsilon
$$

Здесь $\|\cdot\|$ - норма в $L_{2}$. После этого замечания разность (14) следует представить в виде

$$
\begin{aligned}
& \left(U^{t_{1}} g^{\prime}, g^{\prime \prime}\right)-\left(U^{t_{2}} g^{\prime}, g^{\prime \prime}\right)+ \\
& \quad+\left(U^{t_{1}}\left(f^{\prime}-g^{\prime}\right), f^{\prime \prime}-g^{\prime \prime}\right)+\left(U^{t_{1}}\left(f^{\prime}-g^{\prime}\right), g^{\prime \prime}\right)+\left(U^{t_{1}} f^{\prime}, f^{\prime \prime}-g^{\prime \prime}\right)- \\
& \quad-\left(U^{t_{2}}\left(f^{\prime}-g^{\prime}\right), f^{\prime \prime}-g^{\prime \prime}\right)-\left(U^{t_{2}}\left(f^{\prime}-g^{\prime}\right), g^{\prime \prime}\right)-\left(U^{t_{2}} f^{\prime}, f^{\prime \prime}-g^{\prime \prime}\right)
\end{aligned}
$$

Согласно основной лемме, разность в первой строке можно сделать сколь угодно малой при достаточно больших значениях $t_{1}$ и $t_{2}$. Ввиду неравенства Коши-Шварца, унитарности оператора Купмана $U$ и неравенств (15) остальные слагаемые в (16) стремятся к нулю равномерно по $t_{1}$ и $t_{2}$, когда $\varepsilon \rightarrow 0$.

Остальная часть этого параграфа посвящена доказательству основной леммы.

Положим $A_{0}=A^{\prime} \cap A^{\prime \prime}, I_{0}=I^{\prime} \cap I^{\prime \prime}$. Пусть $\chi_{0}: \Lambda \times I \rightarrow \mathbb{R}$ - характеристическая функция (индикатор) измеримого множества $A_{0} \times I_{0}$, а $\tilde{\chi}_{0}: \Lambda \rightarrow \mathbb{R}$-характеристическая функция множества $A_{0}$. Ясно, что

$$
J(t)=\int_{I_{0}}\left(U_{*}^{\alpha(\omega) t} \tilde{\chi}_{0}, \tilde{\chi}_{0}\right) d \sigma
$$

Пусть $D_{\gamma}=\left\{\omega \in I_{0}:\left|\alpha^{\prime}(\omega)\right|>\gamma\right\}, \alpha^{\prime}=d \alpha / d \omega$. Согласно предположению, критические точки функции $\omega \mapsto \alpha(\omega)$ изолированы. Следовательно, $\sigma$-мера множества $I \backslash D_{\gamma}$ стремится к нулю при $\gamma \rightarrow 0$. Более того, можно считать, что $D_{\gamma}$ - объединение конечного числа интервалов. Пусть $\left(\omega_{1}, \omega_{2}\right)$ - один из интервалов, составляющих $D_{\gamma}$. Тогда $\alpha$ можно считать координатой на $\left(\omega_{1}, \omega_{2}\right)$. Действительно, функция $\omega(\alpha)$, обратная к $\alpha:\left(\omega_{1}, \omega_{2}\right) \rightarrow \mathbb{R}$, сушествует и является гладкой. Положим $d \sigma(\omega)=h(\omega) d \omega$. Согласно условиям теоремы 3 функция $\omega \rightarrow h(\omega)$ интегрируема: $h \in L_{1}(I, d \omega)$. Таким образом,

$$
\int_{\omega_{1}}^{\omega_{2}}\left(U_{*}^{\alpha(\omega) t} \tilde{\chi}_{0}, \tilde{\chi}_{0}\right) h(\omega) d \omega=\int_{\alpha\left(\omega_{1}\right)}^{\alpha\left(\omega_{2}\right)}\left(U_{*}^{\alpha t} \tilde{\chi}_{0}, \tilde{\chi}_{0}\right) h(\omega(\alpha)) \omega^{\prime}(\alpha) d \alpha
$$

Так как $h(\omega(\alpha)) \omega^{\prime}(\alpha) \in L_{1}\left(\left(\alpha\left(\omega_{2}\right), \alpha\left(\omega_{1}\right)\right), d \alpha\right)$, то согласно теореме 2 интеграл $(17)$ имеет предел при $t \rightarrow \infty$.

Лемма полностью доказана. 


\section{6. ВОЗРАСТАНИЕ ЭНТРОПИИ}

Как известно, в статистической механике энтропия определяется как интеграл

$$
S_{t}=-\int_{\Gamma} \rho_{t} \ln \rho_{t} d \mu, \quad d \mu=d^{2 n} z .
$$

Так как $\rho_{t}(z)=\rho\left(g^{-t}(z)\right)$ и поток $g^{t}$ сохраняет меру $\mu$, то очевидно, что $S_{t}=$ const. Это замечание является частным случаем общего результата Пуанкаре о постоянстве тонкой энтропии динамических систем [4].

$\mathrm{C}$ другой стороны, $\rho_{t}$ слабо сходится к $\bar{\rho}$ при $t \rightarrow \pm \infty$. Обобщая аргументацию Гиббса и Пуанкаре, можно считать, что стационарная плотность распределения вероятностей $\bar{\rho}$ отвечает тепловому равновесию рассматриваемой динамической системы. Поэтому естественно ввести энтропию равновесного состояния

$$
S_{\infty}=-\int_{\Gamma} \bar{\rho} \ln \bar{\rho} d \mu
$$

Теорема 4. Имеет место равенство

$$
S_{t} \leqslant S_{\infty}
$$

Для доказательства используем свойство вогнутости функции $h(x)=-x \ln x$ при положительных значениях $x$. Так как $S_{t}=$ const, то по теореме Фубини

$$
S_{t}=\frac{1}{T} \int_{0}^{T} \int_{\Gamma} h\left(\rho_{t}\right) d \mu d t=\int_{\Gamma}\left[\frac{1}{T} \int_{0}^{T} h\left(\rho_{t}\right) d t\right] d \mu .
$$

Ввиду неравенства Иенсена (см. [10])

$$
\frac{1}{T} \int_{0}^{T} h\left(\rho_{t}\right) d t \leqslant h\left(\frac{1}{T} \int_{0}^{T} \rho_{t} d t\right), \quad T>0 .
$$

Следовательно,

$$
S_{t} \leqslant \int_{\Gamma} h\left(\frac{1}{T} \int_{0}^{T} \rho_{t} d t\right) d \mu
$$

Переходя к пределу при $T \rightarrow \infty$ и используя теорему 1 , получаем требуемое.

ЗАмЕчАниЕ 3. Для бесстолкновительной среды в прямоугольном параллелепипеде неравенство (18) установлено ранее в работе [11]. В некоторых частных случаях оно отмечено еше Пуанкаре [4].

Поскольку $\rho_{t}$ слабо сходится к одной и той же функции $\bar{\rho}$ как при $t \rightarrow+\infty$, так и при $t \rightarrow-\infty$, то заключение теоремы 4 о возрастании энтропии инвариантно при обрашении времени $t$. Напомним, что из кинетического уравнения Больцмана вытекает монотонное возрастание энтропии при увеличении времени, и с этим связан знаменитый парадокс Лошмидта. Обсуждение этого парадокса с разных точек зрения можно найти, например, в монографиях [12]. 
В заключение сделаем замечание о критике Крыловым [13] (с.51-52) результатов Пуанкаре о возрастании энтропии бесстолкновительной среды при возмущениях [4]. Пуанкаре рассматривает равновесное состояние одномерного идеального газа, равномерно заполняющего некоторый отрезок. Из бесконечности к отрезку приближается гравитируюшее тело, газу дается возможность принять новое равновесное состояние, а затем тело снова удаляют на бесконечное расстояние. В работе [4] вычислено прирашение энтропии, которое оказалось положительным.

Крылов замечает, что этот вывод противоречит результату работы Пуанкаре о постоянстве тонкой энтропии (неавтономность уравнений движения не играет никакой роли). На самом же деле никакого противоречия здесь нет. Для состояний теплового равновесия Пуанкаре фактически заменял (не оговаривая этого явно) начальную плотность вероятности ее слабым пределом. Когда тело приближается к отрезку с газом, то соответствующая динамическая система с одной степенью свободы изменяется: к гамильтониану добавляется потенциальная энергия гравитационного взаимодействия. Слабый предел $\rho_{-}$этой новой нелинейной системы оказывается функцией от полной энергии. Далее тело удаляется, функция $\rho_{-}$становится начальной плотностью распределения, а гамильтониан снова будет совпадать с кинетической энергией. Поэтому слабый предел $\rho_{+}$решения уравнения Лиувилля новой системы с начальным условием $\rho_{-}$становится функцией, зависяшей лишь от кинетической энергии. По теореме 4 $S_{+} \geqslant S_{-}$. На самом деле, как показывают вычисления Пуанкаре, $S_{+}>S_{-}$.

Благодарности. Работа выполнена при финансовой поддержке грантов РФФИ № 02-01-00400, 02-01-01059, 00-15-99269 и INTAS 00-221. Авторы благодарят В. Тена за полезные обсуждения.

\section{Список литературы}

[1] Джс. В. Гиббс. Основные принципы статистической механики. М.: Гостехиздат, 1946.

[2] Н. Н. Боголюбов. Проблема динамической теории в статистической физике. М.-Л.: Гостехиздат, 1946.

[3] Дж. Уленбек, Дж. Форд. Лекции по статистической механике. М.: Мир, 1965.

[4] А. Пуанкаре. Замечания о кинетической теории газов. В сб.: Избранные труды. Т. ІІІ. М.: Наука, 1974. С. 385.

[5] П. Халмош. Лекции по эргодической теории. М.: ИЛ, 1959; A. Lasota, M. C. Mackey. Probabilistic properties of deterministic systems. Cambridge: Cambridge University Press, 1985.

[6] В. В. Козлов. Докл. РАН. 2002. Т. 382. № 5. С. 593

[7] В. В. Немыцкий, В.В. Степанов. Качественная теория дифференциальных уравнений. М.-Л.: Гостехиздат, 1949.

[8] Г. Харди. Расходящиеся ряды. М.: ИЛ, 1951.

[9] Ф. Рисс, Б. Сёкефальви-Надь. Лекции по функциональному анализу. М.: Мир, 1979.

[10] Г. Г. Харди, Д. Е. Литтлвуд, Г. Полиа. Неравенства. М.: ИЛ, 1948.

[11] V. V. Kozlov. Reg. Chaotic Dyn. 2001. V. 6. № 3. P. 235.

[12] М. Кац. Вероятность и смежные вопросы в физике. М.: Мир, 1965; И. Пригожси, И. Стенгерс. Порядок из хаоса. М.: Прогресс, 1986.

[13] Н. С. Крылов. Работы по обоснованию статистической физики. М.-Л.: Изд-во АН СССР, 1950. 